\title{
CRYSTAL PHYSICS
}

$\mathrm{T}$ $\mathrm{HE}$ presentation of the frontier regions of modern physics to an audience with a variable appreciation of scientific and mathematical ideas is, inevitably, a difficult task. None the less, it is one which should not be shunned, unless we are inclined to assume the semantic barrier to be impassable between the pure scientist and the educated public. In the realm of crystal physics with its many facets, the choice of electron-states in crystals as a topic for a discussion by Section A (Physies) of the British Association at the recent meeting in York, did not minimize the difficulties of communication. However, in the first lecture of the session, I attempted to wean the audience from a 'billiard ball' conception of atomic particles and, with little more than de Broglie's hypothesis and the Bragg reflexion law to assist me, to carry them into the mysteries of the energy-band theory of solids. It was not too difficult to begin a discussion of the wave behaviour of electrons in the ordered periodic-field of a crystal lattice, but the going became somewhat harder when the unavoidable introduction of wave vector space occurred. However, the vagaries of the effective electron mass were a little better appreciated by adding the visual aid of a bubble in a spirit-level as an analogy of the positive hole. It was inevitable that such treatment by analogy involved a considerable loss of rigour, but to me it proved to be a valuable exercise in transmitting the important results of the wave mechanical theory of solids without recourse to the mathematical building-materials allowable to the postgraduate seminar room.

The niceties of the band theory for electrons in a perfect crystal were soon disturbed by the reversion to practical situations. The next phase of the lecture dealt with the various crystal imperfections, their effect on electron and hole behaviour and their importance in semiconductor electronics. Again, treating the matter non-rigorously in terms of simple electrostatic forces, brings the problem of donor-and acceptor-levels and types of lattice vacancies into a relatively familiar perspective, and in this instance introduced some of the ideas to be used in the following lecture given by Dr. V. Hesketh.

Many of the important foundations of both experimental and theoretical solid-state physics were laid by the pioneer work of Prof. R. W. Pohl and his research school in Göttingen using the simplest of crystals, the ionic alkali halides. For an audience raised by this stage of the proceedings into the "thin but bracing air' of wave mechanies, the appearance in a place of honour among beautifully coloured single-crystals of a familiar packet of common tablesalt provided a 'down to earth' relief. The theme of Dr. Hesketh's lecture was the alkali halide crystal as a model solid for investigation. Such a claim appears to have been justified by the subsequent discussion. Optical, electrical and other studies provide some of the most certain evidence on the nature of lattice vacancies, single or in aggregation, in solid-state physics to-day. An important feature of this talk was the link which it indicated between such electronic investigations and those concerned with mechanical properties and the role of dislocations in crystals. A connexion with another interest of Section A, magnetism, was in evidence in the paramagnetic-resonance studies of alkali halides. From the general discussion which followed the lecture there was a hint that a 'North eye' was being turned towards dislocations by those, like Prof. L. F. Bates, who follow the motion of magnetic domains. It was a pity that there was no opportunity to demonstrate other links which are rapidly being forged between different branches of solid-state physics and those under examination.

If one might, in conclusion, offer an overall impression of this session in the proceedings of Section $\mathrm{A}$ at the York meeting, it is that the conditions of limited time and contact of the meetings have a very stultifying effect on any attempt to make an effective contribution to the communication of new physical concepts to a wider audience of educated people. On this occasion, in spite of the central position occupied by wave-mechanical ideas in the session, it was not a mathematical but a time barrier which blocked the transmission of information. Perhaps this will be borne in mind by the Association in framing a new position for itself as a most important medium for translation as well as transmission of scientific information. From my own limited experience, there is no doubt that courses extended over the year, making use of the facilities offered by extra-mural departments of the universitios and similar organizations for further education, would provide a means of digestion for the strong meat of modern physics. As a corollary, the lecturer must bend his rigour of thought to breaking point so that he may admit familiar, though inadequate, analogies as carriers across the semantic obstacles.

$$
\text { G. F. J. GarLick }
$$

\section{SCIENCE BY THE UPPER FORM}

$\mathrm{A}$ $\mathrm{N}$ innovation of Section X (Assembly of Corresponding Societies) of the British Association, whereby a selected panel of young scientists of sixth-form status are able to present short papers on projects with which each has been connected, was continued for the fifth successive year at the York meeting of the Association. This year there were five speakers drawn from Yorkshire schools, each one representing a school team engaged on the aequisition of scientific knowledge in the field, under competent leadership.

Ampleforth College, York, led the way with a paper by M. L. M. Wright on "Physiography and Scenery of the Isle of Eigg", excellently illustrated by colour slides showing the rugged grandeur of an isolated area along the western Scottish coast of only some twelve square miles in extent. Life is hard for the humans living there, and precarious for plant and 